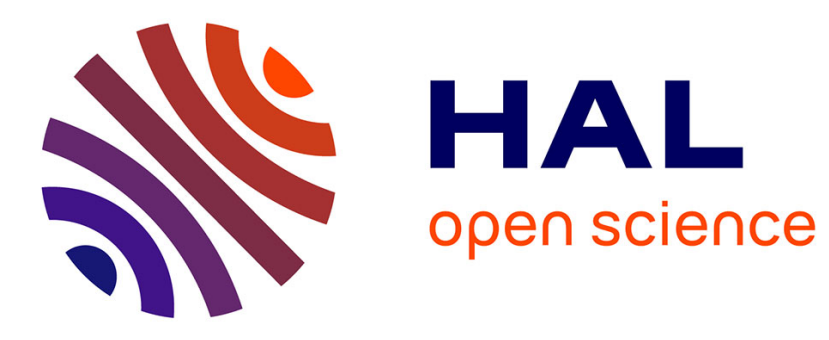

\title{
Correlation between negative magnetoresistance effect and magnon excitations in single-crystalline $\mathrm{CuCr} 1.6 \mathrm{~V} 0.4 \mathrm{Se} 4$
}

Ewa Malicka, Tadeusz Groń, Danuta Skrzypek, Andrzej W. Pacyna, Dariusz Badurski, Alicja Waśkowska, Slawomir Mazur, Rafal Sitko

\section{To cite this version:}

Ewa Malicka, Tadeusz Groń, Danuta Skrzypek, Andrzej W. Pacyna, Dariusz Badurski, et al.. Correlation between negative magnetoresistance effect and magnon excitations in single-crystalline CuCr1.6V0.4Se4. Philosophical Magazine, 2010, 90 (11), pp.1525-1541. 10.1080/14786430903405504 . hal-00584287

\section{HAL Id: hal-00584287 https://hal.science/hal-00584287}

Submitted on 8 Apr 2011

HAL is a multi-disciplinary open access archive for the deposit and dissemination of scientific research documents, whether they are published or not. The documents may come from teaching and research institutions in France or abroad, or from public or private research centers.
L'archive ouverte pluridisciplinaire $\mathbf{H A L}$, est destinée au dépôt et à la diffusion de documents scientifiques de niveau recherche, publiés ou non, émanant des établissements d'enseignement et de recherche français ou étrangers, des laboratoires publics ou privés. 




\section{Correlation between negative magnetoresistance effect and magnon excitations in single-crystalline CuCr1.6V0.4Se4}

\begin{tabular}{|r|l|}
\hline Journal: & Philosophical Magazine \& Philosophical Magazine Letters \\
\hline Manuscript ID: & TPHM-09-Jul-0306 \\
\hline Dournal Selection: & Philosophical Magazine \\
\hline Complete List of Authors: & 20-Jul-2009 \\
\hline & $\begin{array}{l}\text { Malicka, Ewa; University of Silesia, Institute of Chemistry } \\
\text { Groń, Tadeusz; University of Silesia, Institute of Physics } \\
\text { Skrypek, Danuta; University of Silesia, Institute of Physics } \\
\text { Pacyna, Andrzej; Polish Academy of Sciences, The Henryk } \\
\text { Niewodniczański Institute of Nuclear Physics } \\
\text { Badurski, Dariusz; Polish Academy of Sciences, Institute of Low } \\
\text { Temperatures and Structure Research } \\
\text { Waśkowska, Alicja; Polish Academy of Sciences, Institute of Low } \\
\text { Temperatures and Structure Research } \\
\text { Mazur, Sławomir; University of Silesia, Institute of Physics } \\
\text { Sitko, Rafał; University of Silesia, Institute of Chemistry }\end{array}$ \\
\hline Keywords: & $\begin{array}{l}\text { electron paramagnetic resonance, magnetoresistance, spinels, } \\
\text { thermoelectric power }\end{array}$ \\
\hline Keywords (user supplied): & magnetoresistance, spin waves, thermoelectric effects \\
\hline
\end{tabular}

\section{(5cholaroNE \\ Manuscript Central}




\title{
Correlation between negative magnetoresistance effect and magnon excitations in single-crystalline $\mathrm{CuCr}_{1.6} \mathrm{~V}_{0.4} \mathrm{Se}_{4}$
}

\author{
E. Malicka, ${ }^{1}$ T. Groń, ${ }^{2}$ D. Skrzypek, ${ }^{2}$ A.W. Pacyna, ${ }^{3}$ D. Badurski, ${ }^{4}$ \\ A. Waśkowska, ${ }^{4}$ S. Mazur, ${ }^{2}$ and R. Sitko ${ }^{1}$ \\ ${ }^{1}$ University of Silesia, Institute of Chemistry, ul. Szkolna 9, 40-006 Katowice, Poland \\ ${ }^{2}$ University of Silesia, Institute of Physics, ul. Uniwersytecka 4, 40-007 Katowice, Poland \\ ${ }^{3}$ The Henryk Niewodniczański Institute of Nuclear Physics, Polish Academy of Sciences, \\ ul. Radzikowskiego 152, 31-342 Kraków, Poland \\ ${ }^{4}$ Institute of Low Temperature and Structure Research, Polish Academy of Sciences, \\ ul. Okólna 2, 50-950 Wrocław, Poland
}

The structural, electrical and magnetic measurements as well as the electron spin resonance (ESR) spectra were used to characterize the singlecrystalline $\mathrm{CuCr}_{1.6} \mathrm{~V}_{0.4} \mathrm{Se}_{4}$ spinel and to study a correlation between negative magnetoresistance effect and magnon excitations. The ferromagnetic order below the Curie temperature $T_{\mathrm{C}} \approx 193 \mathrm{~K}$, a $p$-type semiconducting behavior, the ESR change from paramagnetic to ferromagnetic resonance at $T_{\mathrm{C}}$, a big value of ESR linewidth and its temperature dependence in paramagnetic region were established. The electrical studies revealed the negative magnetoresistance, which can be enhanced with increasing magnetic field and decreasing temperature while a detailed thermopower analysis showed the magnon excitations at low temperatures. The spin-phonon coupling is explained within a 
framework of the complex model of paramagnetic relaxation processes as the several-stage relaxation process in which the $\mathrm{V}^{3+}$ ions, the exchange subsystem and conduction electrons subsystem act as the intermediate reservoirs.

\begin{abstract}
Keywords: magnetoresistance; spin waves; thermoelectric effects; electron spin resonance
\end{abstract}

\title{
1. Introduction
}

Kanamori [1] predicted that $90^{\circ}$ ferromagnetic (FM) superexchange occurs between octahedrally coordinated $3 d^{3}$ ions. For example, in the p-type FM semiconductor $\mathrm{CdCr}_{2} \mathrm{Se}_{4}$ with the Curie temperature $T_{\mathrm{C}}=142 \mathrm{~K}$ and the CurieWeiss $(\mathrm{CW})$ temperature $\theta_{\mathrm{CW}}=190 \mathrm{~K}$ the FM superexchange occurs within the Cr sublattice via the $90^{\circ} \mathrm{Cr}-\mathrm{Se}-\mathrm{Cr} \sigma / \pi$ bonding which links a half-filled $t_{2 \mathrm{~g}}(\pi)$ orbital of one of Cr-ions with an empty $e_{\mathrm{g}}(\sigma)$ level on the other $\mathrm{Cr}[2,3]$. In the parent $\mathrm{FM} \mathrm{CuCr} 2 \mathrm{Se}_{4}$ being a $p$-type metallic conductor with $T_{\mathrm{C}}=416 \mathrm{~K}$ and $\theta_{\mathrm{CW}}$ $=436 \mathrm{~K}$ [4], the chromium spins are coupled ferromagnetically via double exchange interaction involving the electrons jumping between $\mathrm{Cr}^{3+}$ and $\mathrm{Cr}^{4+}$ ions $[5,6]$. The X-ray photoelectron spectroscopy and polarized neutron diffraction study for $\mathrm{CuCr}_{2} \mathrm{Se}_{4}$ provided a direct evidence for the presence of monovalent copper atoms with a $3 d^{10}$ configuration [7,8]. However, it was not possible to measure in $\mathrm{CuCr}_{2} \mathrm{Se}_{4}$ the magnetic splitting of the $\mathrm{Cr} 3 s$ levels, because these levels overlapped with the $\mathrm{Cu} 3 p$ ones [7]. The second end composition of the 
$\mathrm{CuCr}_{2-\mathrm{x}} \mathrm{V}_{\mathrm{x}} \mathrm{Se}_{4}$ system, i.e $\mathrm{CuV}_{2} \mathrm{Se}_{4}$ is not known, yet. In the isostructural $\mathrm{CuCr}_{2-}$ ${ }_{\mathrm{x}} \mathrm{V}_{\mathrm{x}} \mathrm{S}_{4}$ spinel system a rapid decrease of $T_{\mathrm{C}}$ from $267 \mathrm{~K}$ for $x=0.25$ to $8 \mathrm{~K}$ for $x$ $=1.75$ was observed [9]. This fact has been explained by being weaker Cr-S-V superexchange interactions than the original $\mathrm{Cr}-\mathrm{S}-\mathrm{Cr}$ ones as well by a weakening of the nearest neighbor ferromagnetic $\mathrm{Cr}-\mathrm{S}-\mathrm{Cr}$ interactions due to the initial lattice expansion [9].

Recently, numerous efforts have been made to find a correlation between the thermoelectric power and the double exchange and superexchange interactions in the $\mathrm{CdCr}_{2-\mathrm{x}} \mathrm{Ga}_{\mathrm{x}} \mathrm{Se}_{4}, \mathrm{Zn}_{\mathrm{x}} \mathrm{Cu}_{\mathrm{y}} \mathrm{Cr}_{\mathrm{z}} \mathrm{Se}_{4}, \mathrm{Cu}_{\mathrm{x}} \mathrm{Ga}_{\mathrm{y}} \mathrm{Cr}_{\mathrm{z}} \mathrm{Se}_{4}$ and $\mathrm{Cu}_{\mathrm{x}} \mathrm{Co}_{\mathrm{y}} \mathrm{Cr}_{\mathrm{z}} \mathrm{Se}_{4}$ spinel series [10-14]. These studies have shown that the strong ferromagnetic coupling connected mainly with the double-exchange mechanism, makes easier both the magnon excitations and a transfer of the phonon momentum to the electron gas. The opposite behavior for the ferrimagnetic and antiferromagnetic spin arrangements was observed.

It seems thus natural to expect a coupling between the magnon and electron systems leading to the negative magnetoresistance effect in single-crystalline $\mathrm{CuCr}_{1.6} \mathrm{~V}_{0.4} \mathrm{Se}_{4}$ spinel. For that the Bloembergen and Wang model of paramagnetic relaxation processes [15], the high-temperature expansion of the magnetic susceptibility and the thermoelectric power analysis were used.

\section{Experiment}

\subsection{Preparation}

The single crystal of $\mathrm{CuCr}_{1.6} \mathrm{~V}_{0.4} \mathrm{Se}_{4}$ spinel was obtained by the chemical 
vapour transport method [16] in a closed quartz ampoule containing binary selenide $\mathrm{CuSe}$, and stoichiometric amounts of high purity (99.99\%) elements: vanadium and selenium. Anhydrous chromium chloride $\mathrm{CrCl}_{3}$ was used as the chemical transport agent. The ampoule was heated in a horizontal zone furnace for four weeks. The temperature of the solution and crystallization zone was $1273 \mathrm{~K}$ and $1223 \mathrm{~K}$, respectively. The resulting single crystal was of octahedral shape with regular $\{111\}$ faces, the edge length being $4 \mathrm{~mm}$.

For the crystal structure characterization, describing the cation distribution over the tetrahedral and octahedral sites formed by the selenium sublattice, a good quality sample was selected for the intensity data collection with a single crystal X-ray diffraction technique. The intensities were measured with a KM4/CCD (Oxford Diffraction) instrument, operating in $\kappa$ geometry and using graphite monochromated MoK $\alpha$ radiation $(\lambda=0.71073 \AA)$. The $\omega$-scan technique was used for data collection. Integration of the intensity data and corrections for Lorentz - polarization effects and absorption were made using CrysAlis software [17]. The absorption correction was applied with the Gaussian face-indexed numerical routine [18]. The structure calculations were performed using the SHELXL-97 program system [18].

The chemical composition of the $\mathrm{CuCr}_{1.6} \mathrm{~V}_{0.4} \mathrm{Se}_{4}$ single crystal was determined by the energy-dispersive X-ray spectrometry (EDXRF). The sample was excited by the air-cooled side-window Rh target X-ray tube of $125 \mu \mathrm{m}$ thickness Be window and ca. $100 \mu \mathrm{m}$ nominal focal spot size (XTF 5011/75, Oxford Instruments, USA). The X-ray tube was supplied with the XLG high- 
voltage generator (Spellman, USA). The tungsten pinhole collimator was used to reduce the size of analyzed area. For the collimators of the size holes of 100 , 200, 400, 1000 and $2000 \mu \mathrm{m}$, the following focal spot sizes are obtained: 169, $280,581,1469,2888 \mu \mathrm{m}$. The X-ray spectra of the samples were collected by thermoelectrically cooled Si-PIN detector (XR-100CR Amptek, Bedford, MA, USA) of $6 \mathrm{~mm}^{2}$ active area, $500 \mu \mathrm{m}$ crystal thickness and $12.5 \mu \mathrm{m}$ Be window thickness. The Si-PIN detector cooled to the temperature of ca. $-55^{\circ} \mathrm{C}$ reaches the resolution of $145 \mathrm{eV}$ at $5.9 \mathrm{keV}$. The $\mathrm{Si}$-PIN detector was coupled to a multichannel analyzer (PX4 Amptek, Bedford, MA, USA). In the constructed spectrometer, the incidence and take-off angles were $45^{\circ}$. The position of the sample was moved using the X-Y stage and was monitored by CCD camera and two laser pointers. The weight composition of the single crystal under study is as follows: $\mathrm{Cu}-13.1 \pm 0.2 \mathrm{Cr}-17.3 \pm 0.3, \mathrm{~V}-4.26 \pm 0.08, \mathrm{Se}-65.4 \pm 0.8$. These values have been converted into percent of the atomic content.

\subsection{Electrical and magnetic measurements}

The mass magnetization was measured at $4.6 \mathrm{~K}$ and in external magnetic fields up to $60 \mathrm{kOe}$ while the static (dc) mass susceptibility measurements were performed in the temperature range $4.2-350 \mathrm{~K}$ and in applied external magnetic field of 900 Oe using a Faraday type Cahn RG automatic electrobalance.

The electrical measurements have been done in the temperature range 7$300 \mathrm{~K}$. The electrical resistivity $\rho$ has been measured with the 4-point dc method using the apparatus with Keithley K181 digital multimeters. The maximal error 
$\delta \rho / \rho$ was less than $\pm 1 \%$. The magnetoresistance measurements were performed in the magnetic fields up to $8 \mathrm{~T}$ at $4.3,10,15,20$ and $40 \mathrm{~K}$ and at fixed magnetic field $8 \mathrm{~T}$ in the temperature range $7-110 \mathrm{~K}$. The direction of magnetic field was perpendicular to the current sample. The thermoelectric power was measured with a differential method using the temperature gradient $\Delta T$ of about $2 \mathrm{~K}$. The accuracy of the value of thermopower was estimated to be better than $3 \mu \mathrm{V} / \mathrm{K}$. The electrical and thermal contacts between the single crystal and the copper rods were maintained with a silver lacquer mixture (Degussa Leitsilber 200). The thermoelectric power and the electrical resistivity of the same sample were measured along the $<001>$ direction. The crystal orientation about the $<001>$ crystallographic zone axis, was based on 12-18 centered X-ray diffraction reflections with the Bragg angles in the range $107 \leq 2 \theta \leq 144^{\circ}$ (MoK $\alpha$ radiation). The checking electrical measurements in the $<111>$ direction, performed for comparison, showed unimportant difference with those along $<001>$.

\subsection{ESR measurements}

The electron spin resonance (ESR) spectra were measured in the temperature range $77-275 \mathrm{~K}$ using a $100-\mathrm{kHz}$ field-modulated spectrometer operated at $9 \mathrm{GHz}$, so that the derivatives of the absorption signals were recorded. A continuous nitrogen flow cryostat was used to study temperature dependence. For ESR measurements the obtained crystals were crushed into powder and diluted in the paraffin to reduce the skin effect. The shape and area 
of the ESR spectra were analyzed by numerical method.

\section{Results}

\subsection{Crystallographic properties}

Crystal data, experimental details and structure refinement results for the single-crystal of $\mathrm{CuCr}_{1.6} \mathrm{~V}_{0.4} \mathrm{Se}_{4}$ spinel are collected in Table 1. They are showing that the compound crystallizes in a cubic structure with space group $F d \overline{3} m$ (\#227) and the unit cell of dimension $a=1034.99(12)$ pm, being slightly elongated in comparison with $a=1033.5(3) \mathrm{pm}$ of the parent $\mathrm{CuCr}_{2} \mathrm{Se}_{4}$ [6]. The structure model with the diamagnetic $\mathrm{Cu}$ ions occupying the tetrahedral $8 a$ positions and the magnetic $\mathrm{Cr}$ and $\mathrm{V}$-ions sharing the octahedral $16 d$ sites, converged satisfactorily. The site occupancy factors treated as free variables in the least-squares structure refinement, confirmed the sample composition and the cation distribution according to the spinel formula: $\mathrm{Cu}\left[\mathrm{Cr}_{1.6} \mathrm{~V}_{0.4}\right] \mathrm{Se}_{4}$.

\subsection{Electrical and magnetic properties}

The electrical resistivity $\rho(\mathrm{T})$ and thermoelectric power $S(\mathrm{~T})$ measurements depicted in Fig. 1 reveal a $p$-type semiconducting behaviour of the $\mathrm{CuCr}_{1.6} \mathrm{~V}_{0.4} \mathrm{Se}_{4}$ single crystal. The values of $\rho(\mathrm{T})$ and $S(\mathrm{~T})$ are close to those for $\mathrm{CuCr}_{2} \mathrm{Se}_{4}$ [19]. The variation of $\rho(\mathrm{T})$ does not follow a simple exponential behaviour, because a gradual decrease of resistivity and strong slope at $T_{\mathrm{C}}$ with increasing temperature are observed. The $S(\mathrm{~T})$ shows a broad hump at $100 \mathrm{~K}$ and reaches a minimum at $T_{\mathrm{C}}$ from one side and goes to $(0,0)$ from the other. 
The $\rho(\mathrm{T})$ dependence decreases with increasing magnetic field, revealing the negative magnetoresistance effect shown in Fig. 2. Moreover, the lower temperature, the larger negative magnetoresistance, $\Delta \rho / \rho_{0}(\mathrm{H})$. However, in the temperature range $10-20 \mathrm{~K}$ the negative values of $\Delta \rho / \rho_{0}(\mathrm{H})$ increase insignificantly as temperature decreases (Fig. 2).

The results of the magnetic measurements are depicted in Figs. 3 and 4. The dc mass susceptibility vs. temperature, $\chi_{\sigma}(\mathrm{T})$, shows a ferromagnetic order with the Curie temperature $T_{\mathrm{C}}=193.2 \mathrm{~K}$. Above $200 \mathrm{~K}, \chi_{\sigma}^{-1}(\mathrm{~T})$ follows the $\mathrm{CW}$ behavior with a positive $\mathrm{CW}$ temperature $\theta_{\mathrm{CW}}=207 \mathrm{~K}$ and an effective moment $\mu_{\text {eff }}=2.83 \sqrt{M C_{\sigma}}=5.85 \mu_{\mathrm{B}} /$ f.u., where the molar mass $M \approx 482.96 \mathrm{~g} / \mathrm{mol}$ and the Curie constant $C_{\sigma}=8.873 \cdot 10^{-3} \mathrm{~K} \cdot \mathrm{cm}^{3} / \mathrm{g}$. This value is somewhat higher than the spin-only value of $5.22 \mu_{\mathrm{B}} /$ f.u. for $3 d^{3} \mathrm{Cr}^{3+}$ and $3 d^{2} \mathrm{~V}^{3+}$ ions as well as of $5.50 \mu_{\mathrm{B}} /$ f.u. for $3 d^{9} \mathrm{Cu}^{2+}, 3 d^{3} \mathrm{Cr}^{3+}$ and $3 d^{2} \mathrm{~V}^{3+}$ ions. It means that the chromium ions may form a mixed valence band. In Fig. 4 a shape of magnetic isotherm, $\sigma(\mathrm{H})$, measured up to $60 \mathrm{kOe}$ and at $4.6 \mathrm{~K}$ exhibits a saturation magnetization of $53.84 \mathrm{Oe} \cdot \mathrm{cm}^{3} / \mathrm{g}\left(4.66 \mu_{\mathrm{B}}\right)$ at $4 \mathrm{kOe}$ already. In the inset of Fig. 4 a linear increase of magnetization with increasing magnetic field, indicates a lack of spontaneous magnetization below 4 kOe.

\subsection{ESR spectra}

The resonance spectra for $\mathrm{CuCr}_{1.6} \mathrm{~V}_{0.4} \mathrm{Se}_{4}$ consist of a single line in the range from room temperature to $T_{1}=200 \mathrm{~K}$. At room temperature the intensity 
of the resonance line is very weak. Below $T_{1}$ the spectra were simulated with two profiles. The Figures 5(a), (b) and (c) show the temperature dependence of the ESR parameters: $\Delta B$ - linewidth, $B_{\mathrm{r}}-$ resonance field and $D I-$ intensity of the spectrum. The corresponding values of the $\Delta B$ and $B_{\mathrm{r}}$ were obtained as the fitting parameters and the $D I$ values were calculated as double integration of the spectrum. Starting from the $T=272 \mathrm{~K}(\Delta B=159.2 \mathrm{mT})$ the linewidth values rapidly decrease as the temperature is lowered to $T_{1}$ where $\Delta B=27.8 \mathrm{mT}$. Next, the linewidth passes through a minimum and with decreasing temperature shows a continuous broadening. The line-shape analysis shows that the ESR line remains Lorentzian down to $T=212 \mathrm{~K}$, and below this temperature a deviation towards an asymmetrical line occurs. The shift of the resonance field $\left(B_{\mathrm{r}}\right)$ from the high temperature value is observed below $T=212 \mathrm{~K}$. In the temperature range from $T=193 \mathrm{~K}$ to $T=85 \mathrm{~K}$ this effect is very significant. In Fig. 5(c) the temperature evolution of the intensity of the ESR spectrum is shown with the values reduced to $T=272 \mathrm{~K}$. In the temperature range from $T=225 \mathrm{~K}$ to $T=$ $183 \mathrm{~K}$, the intensity rapidly increases. The intensity of ESR spectrum defined as $D I$ (double integration of the spectrum) should be proportional to the spin susceptibility of the sample. The Curie temperature estimated from the DI(T) dependence equals 193.8 K. The agreement with the susceptibility measurements is satisfactory thus the ESR data appear to be consistent with the magnetic ones. 


\section{Discussion}

An explanation of the negative magnetoresistance effect in singlecrystalline $\mathrm{CuCr}_{1.6} \mathrm{~V}_{0.4} \mathrm{Se}_{4}$ spinel is considered within a framework of spin-spin and spin-phonon couplings and also as a coupling between magnon and electron systems. For that a complex model of the paramagnetic relaxation processes and the thermoelectric power analysis are discussed in detail.

\subsection{ESR studies}

The linewidth value of $\mathrm{CuCr}_{1.6} \mathrm{~V}_{0.4} \mathrm{Se}_{4}$ at room temperature is about $200 \mathrm{mT}$. To understand the origin of the observed linewidth we have to estimate the relaxation contributions of the relevant spin-spin and spin-phonon interactions. In the chromium spinels, the $\mathrm{Cr}^{3+}$ ions always occupy the $\mathrm{B}$-site of the spinel structure. The local symmetry at the octahedral site leads to the nondegenerate orbital ground state with $S=3 / 2$. On the other hand, for the $3 d^{2}$ configuration of $\mathrm{V}^{3+}(S=1)$, the ${ }^{3} F$ free ion ground state is split by the octahedral field leaving an orbital triplet $\left(T_{2}\right)$ lowest. Because of that, $\mathrm{Cr}^{3+}$ is the ion weakly coupled to the lattice in comparison with the fast-relaxing $\mathrm{V}^{3+}$ ions [19].

The ESR linewidth, $\Delta B$, is related to the relaxation of the spin system. For individual spins $\Delta B \sim 1 / \tau$, where $\tau$ is the spin relaxation time. In a dense magnetic material, this relationship is modified since the magnetization relaxes towards an effective field instead of the external field. In the examined compound it can be expected that the presence of $\mathrm{V}^{3+}$ ions essentially changes the resonance linewidth. In paramagnetic state (PM) (i.e. for $T>212 \mathrm{~K}$ ) the 
linewidth can be described by the following expression:

$$
\Delta B=\Delta \mathrm{B}_{\mathrm{ss}}+\Delta \mathrm{B}_{\mathrm{s}-\mathrm{ph}}
$$

where $\Delta B_{\mathrm{ss}}$ is described by the exchange narrowing theory [20]:

$$
\Delta B_{\mathrm{ss}}=\left[\left(\Delta \mathrm{B}_{\mathrm{dd}}\right)^{2}\right] / \mathrm{B}_{\mathrm{ex}}
$$

(i.e. is proportional to the square of the dipolar produced linewidth $\Delta B_{\mathrm{dd}}$ divided by the rate of exchange $\left.B_{\mathrm{ex}}\right)$ and $\Delta B_{\mathrm{s}-\mathrm{ph}}$ represents the contributions of the spinphonon interaction.

The complex model of paramagnetic relaxation processes, illustrated in Fig. 6, can be derived by analogy with the model of Bloembergen and Wang $[15,21]$. The Zeeman subsystem contains $\mathrm{Cr}^{3+}$ and $\mathrm{V}^{3+}$ ions and the vanadium is directly coupled to the lattice. The exchange subsystem includes the exchangecoupled $\mathrm{Cr}-\mathrm{Cr}, \mathrm{Cr}-\mathrm{V}, \mathrm{V}-\mathrm{V}$ pairs. In the parent $\mathrm{CuCr}_{2} \mathrm{Se}_{4}$ spinel, which is a well studied metallic ferromagnet with $T_{\mathrm{C}}=416 \mathrm{~K}$ [4], the high value of $T_{\mathrm{C}}$ is related to the exchange through the current of the hole carriers $[5,6]$ including also the subsystem of conduction electrons. That model explains also a big ESR linewidth for $\mathrm{CuCr}_{1.6} \mathrm{~V}_{0.4} \mathrm{Se}_{4}$ and its temperature dependence in the PM state (Fig. 5(a)). The parameter of the thermal broadening linewidth $b=d(\Delta \mathrm{B}) / d \mathrm{~T}=$ $1.9 \mathrm{mT} / \mathrm{K}$ is rather high and means a fast energy flow from the spin subsystem to the $\mathrm{CuCr}_{1.6} \mathrm{~V}_{0.4} \mathrm{Se}_{4}$ lattice [22]. We suppose that the process of spin-lattice 
relaxation includes both the interaction between the conduction electron spins and magnetic spins (Korringa effect) and also the vibrational modulation of exchange interactions. According to the Huber's and Seehra's theory for magnetically concentrated systems $[23,24]$, the relationship between temperature and resonance linewidth can be explained by the Korringa mechanism [22] and spin-phonon relaxation processes in which are participating broad bands of phonons.

The temperature dependence of $\Delta B$ and $B_{\mathrm{r}}$ in the temperature range $212-$ $193 \mathrm{~K}$ (Fig. 5(a) and (b)) indicates that the magnetic short-range order develops. In this case, the spin correlations act as an effective internal field and therefore cause a shift of the resonance field $B_{\mathrm{r}}$ from that at high temperatures, where no magnetic short-range order exists. Simultaneously, the linewidth $\Delta B$ as $T \rightarrow T_{\mathrm{C}}$ from the high temperature side can be written as follows:

$$
\Delta B=\Delta \mathrm{B}_{\text {noncrit }}(\mathrm{T})+\Delta \mathrm{B}_{\text {crit }}(\mathrm{T})
$$

where: $\Delta B_{\text {noncrit }}$ and $\Delta B_{\text {crit }}$ factors represent the noncritical and critical contributions to the linewidth, respectively, and $\Delta B_{\text {noncrit }}$ is given by Eq. (1). The growth of linewidth $\left(\Delta B_{\text {crit }}\right)$ reflects the increase in the lifetime and the correlation length of the critical fluctuations. The appearance of the short-range magnetic ordering is confirmed by the temperature dependence of the spectrum intensity (Fig. 5(c)). As the temperature is reduced from about $T=225 \mathrm{~K}$, the values for intensity rapidly increase. The ferromagnetic ordering temperature $T_{\mathrm{C}}$ 
$=193.8 \mathrm{~K}$ can be inferred from the location of coincident anomalies in the ESR data: $\Delta B, B_{\mathrm{r}}, D I$ (Fig. 5). The ESR spectrum of $\mathrm{CuCr}_{1.6} \mathrm{~V}_{0.4} \mathrm{Se}_{4}$ observed below $T$ $=193 \mathrm{~K}$ undergoes qualitative changes and transforms from paramagnetic (EPR) into ferromagnetic (FMR) resonance. At low temperatures the experimental spectrum was fitted by two profiles shown in Fig. 5(a) and (b). It is known, that FMR can be adequately described in terms of the Landau-Lifshitz-Gilbert equation [25] for the effective internal magnetic fields, which are attributed to demagnetization and magnetocrystalline anisotropy fields. However, as in our resonance measurements the sample in powdered form was examined, the shape of FMR spectrum is the envelop of several components. As can be seen from Fig. 5(a) and (b), the position and linewidth of this spectrum depends monotonously on the temperature.

\subsection{Thermoelectric power analysis}

In this section we consider a coupling between the magnon and electron systems based on the thermoelectric power analysis. Generally, in the magnetic materials the magnetic component of thermopower (MCTP) may result from a coupling of the phonon system to the electron gas via the spin lattice (magnon gas), which is known as the Kasuya model [26], or from the transfer of the magnon momentum to the electron gas (magnon drag) [27]. This magnetic contribution is described by the Bloch law [28], i.e. the spontaneous magnetization decreases with temperature at $T^{3 / 2}$ when $T$ is small [29]. Analogous temperature dependence of MCTP has been directly derived from a 
theoretical model of magnon-assisted transport in a mesoscopic tunnel junction between a ferromagnetic metal and a normal (nonmagnetic) metal [30] for a magnetic component of thermoelectric power.

The experimental curve shape of thermopower $S(T)$ of the spinel single crystal under study (Fig. 1) can be easily fitted by the modified Matoba, Anzai and Fujimori semiempirical formula [31] including the magnetic contribution [13] as follows:

$$
S(T)=D \cdot T+E \cdot T^{3}+\frac{F \cdot\left(\frac{T}{\theta_{D}}\right)^{3}}{G+\left(\frac{T}{\theta_{D}}\right)^{4}}+H \cdot T^{1 / 2}+\frac{I \cdot\left(\frac{T}{J_{e f f}}\right)^{3 / 2}}{K+\left(\frac{T}{J_{e f f}^{a a t}}\right)^{5 / 2}}
$$

where $\theta_{\mathrm{D}}$ is the Debye temperature (see Appendix A), and $J_{e f f}^{a a}$ is the effective exchange integral for the first coordination sphere (see Appendix B). First and second terms in Eq. (4) is the diffusion component where the small correction $E$ is the temperature dependence of $D$ [32], third term in Eq. (4) is the phonon drag component, fourth term in Eq. (4) is the impurity component, and the last term in Eq. (4) is the magnon drag component. $D, E, F, G, H, I$ and $K$ in Eq. (4) (listed in Table II) are the curve-fitting parameters. The agreement index $R$ between the experimental $\left(S_{\text {exp }}\right)$ and the theoretical $\left(S_{\text {theor }}\right)$ thermopowers is high (over $99 \%$, see Table 2).

The results of thermopower analysis of the single-crystalline $\mathrm{CuCr}_{1.6} \mathrm{~V}_{0.4} \mathrm{Se}_{4}$ depicted in Fig. 7 show that the intensity of the magnon drag component $\left(S_{\mathrm{mag}}\right)$ is the largest and reaches a maximum of $7.86 \mu \mathrm{V} / \mathrm{K}$ at $33 \mathrm{~K}$. It means that the magnon excitations, usually driven by the double exchange mechanism, are observed only at low temperatures, and the maximum transfer of the magnon 
momentum to the electron gas may be a main reason for the observed the negative magnetoresistance effect. This effect disappears at higher temperatures. The contribution to the total thermopower originates then mainly from the phonon component $\left(S_{\mathrm{ph}}\right)$ which reveals a maximum of $7.1 \mu \mathrm{V} / \mathrm{K}$ at $100 \mathrm{~K}$, i.e. exactly in the theoretical temperature range $\theta_{\mathrm{D}} / 10-\theta_{\mathrm{D}} / 2$ predicted by the Debye theory [27], where in our case $\theta_{\mathrm{D}}=279.7 \mathrm{~K}$ (see Table 2). At high temperatures the diffusion component $\left(S_{\text {diff }}\right)$ dominates, and according to the Mott formula [33] it is proportional to temperature. The $S_{\text {diff }}=5.6 \mu \mathrm{V} / \mathrm{K}$ at $300 \mathrm{~K}$. The socalled impurity component of the thermopower $\left(S_{\text {imp }}\right)$ described by the $T^{1 / 2}$ law [34] generates a maximum $1.51 \mu \mathrm{V} / \mathrm{K}$ at $300 \mathrm{~K}$.

From the results of the magnetic considerations presented in Table 3 (see Appendix B) it follows that with increasing vanadium content $x$ : (1) contribution of the superexchange interaction to the Curie-Weiss temperature $\theta_{\mathrm{CW}}$ suddenly increases from $90 \mathrm{~K}$ for $\mathrm{CuCr}_{2} \mathrm{Se}_{4}$ to $163 \mathrm{~K}$ for $\mathrm{CuCr}_{1.6} \mathrm{~V}_{0.4} \mathrm{Se}_{4}$, (2) contribution of the double exchange interaction to the Curie-Weiss temperature $\theta_{\mathrm{CW}}$ and the total hopping integral $B$ rapidly decrease, and (3) the $W_{d}$ bandwidth of the $3 d t_{2 g}$ band due to $\mathrm{Cr}^{3+}$ and $\mathrm{Cr}^{4+}$ ions decreases from $0.76 \mathrm{eV}$ for $\mathrm{CuCr}_{2} \mathrm{Se}_{4}$ to $0.11 \mathrm{eV}$ for $\mathrm{CuCr}_{1.6} \mathrm{~V}_{0.4} \mathrm{Se}_{4}$. In the studied single crystal the superexchange $J_{a a}$ and $J_{a b}$ integrals for the first two coordination spheres are positive and negative, respectively, the absolute value of $J_{a a}$ is over nine times larger than $J_{a b}$, the double exchange $b_{a a}$ and $b_{a b}$ integrals for the first two coordination spheres are positive and $b_{a a}$ is five times larger than $b_{a b}$, and the effective exchange 
constants $J_{e f f}^{a a}$ and $J_{e f f}^{a b}$ for the first two coordination spheres are positive and negative, respectively, and the absolute value of $J_{e f f}^{a a}$ is more than five times larger than $J_{e f f}^{a b}$. The calculated magnetic parameters above mentioned well correlate with semiconducting properties and strongly reduced both the long and short range $\mathrm{FM}$ interactions of the $\mathrm{CuCr}_{1.6} \mathrm{~V}_{0.4} \mathrm{Se}_{4}$ spinel. For comparison, the relevant calculated magnetic parameters [13] of the parent $\mathrm{CuCr}_{2} \mathrm{Se}_{4}$ metallic ferromagnet [5] are collected in Table 3.

\section{Conclusions}

We have shown that in ferromagnetic semiconductor $\mathrm{CuCr}_{1.6} \mathrm{~V}_{0.4} \mathrm{Se}_{4}$, exhibiting high electrical conductivity the magnon excitations are connected with a double exchange mechanism existing apart from the superexchange interaction, like it was observed in the ferromagnetic spinel systems: $\mathrm{Zn}_{\mathrm{x}} \mathrm{Cu}_{\mathrm{y}} \mathrm{Cr}_{\mathrm{z}} \mathrm{Se}_{4}$ for $y \geq 0.8$ [11], $\mathrm{Cu}_{\mathrm{x}} \mathrm{Ga}_{\mathrm{y}} \mathrm{Cr}_{\mathrm{z}} \mathrm{Se}_{4}$ for $0.036 \leq y \leq 0.394$ [12], $\mathrm{Cu}_{\mathrm{x}} \mathrm{Co}_{\mathrm{y}} \mathrm{Cr}_{\mathrm{z}} \mathrm{Se}_{4}$ for $0.06 \leq y \leq 0.11$ [13], and indicated by de Gennes in manganites [35]. For comparison, in the $\mathrm{CdCr}_{2-\mathrm{x}} \mathrm{Ga}_{\mathrm{x}} \mathrm{Se}_{4}$ spinel system [10], the ferromagnetic coupling, being caused only by the superexchange mechanism, does not contribute to the spin wave excitations. The big value of ESR linewidth of $\mathrm{CuCr}_{1.6} \mathrm{~V}_{0.4} \mathrm{Se}_{4}$ and its strong temperature increase in PM state suggest the weakly exchange-coupled $\mathrm{Cr}-\mathrm{Cr}$ pairs to the lattice in comparison with the fastrelaxing $\mathrm{V}^{3+}$ ions. The spin-phonon coupling is explained as the several-stage relaxation process in which the $\mathrm{V}^{3+}$ ions, the exchange subsystem and 
conduction electrons subsystem act as the intermediate reservoirs.

Our results mean that in the mixed $\mathrm{Cr}$ valence band with a narrow bandwidth of $0.11 \mathrm{eV}$ a coherent transfer of the magnon momentum to the electron gas results in the negative magnetoresistance effect in the single crystal under study.

\section{Acknowledgments}

This work was partly supported by Ministry of Science (Poland) and funded from science resources for years 2007-2009 as a research project (Project No. N N204 178433). We are indebted to the European Community for the UPGOW fellowship awarded to S. Mazur for the year 2008-09.

\section{Appendix A}

The Debye temperature [36], $\theta_{\mathrm{D}}$, has been estimated from the following formula:

$\theta_{\mathrm{D}}=\frac{\mathrm{hv}}{2 \mathrm{k}} \sqrt[3]{\frac{6 \pi^{2} \mathrm{~N}}{\mathrm{~V}}}$

where $h$ is the Planck constant, $k$ is the Boltzmann constant, $N=56$ is the number of atoms in the spinel unit cell, $V\left(=a^{3}\right.$, where $a$ is the lattice parameter) is the volume of the spinel unit cell and $v=809 \mathrm{~m} / \mathrm{s}$ is the sound speed in the $\mathrm{CuCr}_{2} \mathrm{Se}_{4}$ spinel calculated from Eq. (A1) and based on the value of $\theta_{\mathrm{D}}=280 \mathrm{~K}$ estimated from the heat capacity measurements and the Grüneisen constant. ${ }^{37}$ We assumed the same value of the sound speed in the $\mathrm{CuCr}_{1.6} \mathrm{~V}_{0.4} \mathrm{Se}_{4}$ spinel and 
the calculated value of $\theta_{\mathrm{D}}$ for this spinel is of the same order as for the $\mathrm{CuCr}_{2} \mathrm{Se}_{4}$ one (see Table 2).

\section{Appendix B}

To determine the exchange constants of the superexchange and double exchange interactions an estimation of the $\mathrm{Cr}^{3+}$ and $\mathrm{Cr}^{4+}$ portions is necessary. Portions $x_{3}$ and $x_{4}$ of the chromium ions: $\mathrm{Cr}^{3+}$ and $\mathrm{Cr}^{4+}$, respectively, were calculated from the saturation magnetization $M_{s}$ :

$M_{\mathrm{s}}=2\left(\mathrm{~g}_{3} \mathrm{~S}_{3} \mathrm{x}_{3}+\mathrm{g}_{4} \mathrm{~S}_{4} \mathrm{x}_{4}+\mathrm{g}_{4} \mathrm{~S}_{4} \mathrm{x}\right)$

taking into account the following normalization condition: $x_{3}+x_{4}+x=1$, where $M_{\mathrm{s}}=4.66 \mu_{\mathrm{B}}$ is the experimental value of the saturation magnetization, $S_{3}=3 / 2$ is the spin of $\mathrm{Cr}^{3+}$ ion, $S_{4}=1$ is the spin of $\mathrm{Cr}^{4+}$ and $\mathrm{V}^{3+}$ ions, $g_{3}=2.0$ and $g_{4}=$ 1.86 are the Landé factors for $\mathrm{Cr}^{3+}, \mathrm{Cr}^{4+}$ and $\mathrm{V}^{3+}$ ions, respectively, and $x=0.2$ is the normalized content of $\mathrm{V}^{3+}$ ions.

The exchange constants of superexchange and double exchange interactions between the first two coordination spheres were calculated using the exchange Hamiltonian as follows:

$H=\mathrm{H}_{\mathrm{se}}+\mathrm{H}_{\mathrm{de}}$

where:

$$
H_{s e}=-\sum_{\substack{i, j \\ i \neq j}} J_{i j} \vec{S}_{i} \cdot \vec{S}_{j},
$$

represents the superexchange $\mathrm{Cr}^{\mathrm{n}+}-\mathrm{Se}-\mathrm{Cr}^{\mathrm{m}+}$ and $\mathrm{Cr}^{\mathrm{m}+}-\mathrm{Se}-\mathrm{Se}-\mathrm{Cr}^{\mathrm{n}+}$ interactions [4] with the integrals $J_{\mathrm{ij}}$ and $c_{\mathrm{aa}}=6$ and $c_{\mathrm{ab}}=12$ numbers of nearest neighbours in 
the first and second $\mathrm{Cr}-\mathrm{Cr}$ coordination spheres, respectively. The spins $\vec{S}_{i}$ and $\vec{S}_{j}$ are considered as the quasiclassical vectors.

$$
H_{d e}=-\frac{1}{6} x_{3} x_{4} \sum_{\substack{i, j \\ i \neq j}} b_{i j} \vec{S}_{i} \cdot \vec{S}_{j}
$$

represents the double exchange interactions [4] with $x_{3}$ and $x_{4}$ being the concentration of $\mathrm{Cr}^{3+}$ and $\mathrm{Cr}^{4+}$ ions, which is normalized in such way that $x_{3}+x_{4}$ $+x=1$ as from the ESR studies it follows that $\mathrm{V}^{3+}$ ions are directly coupled to the lattice, and $b_{\mathrm{ij}}$ is the hopping integral between the atomic $t_{2 \mathrm{~g}}$ states $|i\rangle$ and $|j\rangle$ for neighbours $\langle i, j\rangle$. We take the band factor as $\sim x_{3} x_{4}$ corresponding to probability of finding one atom in the state $\mathrm{Cr}^{3+}$ and the neighbouring one in the state $\mathrm{Cr}^{4+}$. The number $1 / 6$ in the double exchange Hamiltonian (B4) is a constant of a bilinear term $\vec{S}_{i} \cdot \vec{S}_{j}$ in the case of spin 1 [38]. Additionally, we take the hopping electron between two $\mathrm{Cr}$ ions on the same footing as the remaining ones. Because all $t_{2 \mathrm{~g}}$ electrons are quantum mechanically indistinguishable, the Cr cations supply both localized and itinerant $3 d$ moments when $\mathrm{Cr}^{4+}$ cations are present [4].

According to the Hamiltonian (B2) the effective exchange constants [4] for the first two coordination spheres are:

$$
\begin{aligned}
& J_{e f f}^{a a}=J_{a a} c_{a a}+\frac{1}{6} b_{a a} c_{a a} x_{3} x_{4}, \\
& J_{e f f}^{a b}=J_{a b} c_{a b}+\frac{1}{6} b_{a b} c_{a b} x_{3} x_{4} .
\end{aligned}
$$

The $J_{\mathrm{aa}}$ and $b_{\mathrm{aa}}$ and $J_{\mathrm{ab}}$ and $b_{\mathrm{ab}}$ integrals for the first two coordination spheres, respectively, are calculated from the high temperature expansion of the magnetic susceptibility [4]. These calculations are based on the equations describing the 
contributions to the paramagnetic Curie-Weiss temperature resulting from the separate mechanisms of the superexchange and double exchange interactions as follows [39]:

$\theta_{C W}=\theta_{\mathrm{se}}+\theta_{\mathrm{de}}$

where $\theta_{\mathrm{CW}}$ is the paramagnetic Curie-Weiss temperature (from experiment), $\theta_{\mathrm{se}}$ is the superexchange contribution to $\theta_{\mathrm{CW}}$ and $\theta_{\mathrm{de}}$ is the double exchange contribution to $\theta_{\mathrm{CW}}$. The above contributions $[4,39]$ can be read:

$\theta_{s e}=\frac{2}{3} X \sum_{p} J_{p} c_{p}$

$\theta_{d e}=\frac{1}{6} X x_{3} x_{4} \sum_{p} b_{p} c_{p}$

where $\sum_{p} J_{p} c_{p}=\theta_{C u}$ was taken from Ref. 4 and equal to $90 \mathrm{~K}$ for the $\mathrm{CuCr}_{2} \mathrm{Se}_{4}$ metallic ferromagnet, which means only the pure superexchange contribution, $B=\sum_{p} b_{p} c_{p}$ is the total hopping integral for the first and second coordination spheres and $X$ is a mixture of spins due to the presence of $\mathrm{Cr}^{3+}, \mathrm{Cr}^{4+}$ and $\mathrm{V}^{3+}$ ions. A summation over $p$ means the summation over the consecutive coordination spheres, each of them containing $c_{\mathrm{p}}$ neighbours of a given central atom. We should set:

$X=\mathrm{x}_{3} \mathrm{~S}_{3}\left(\mathrm{~S}_{3}+1\right)+\mathrm{x}_{4} \mathrm{~S}_{4}\left(\mathrm{~S}_{4}+1\right)+\mathrm{xS}_{4}\left(\mathrm{~S}_{4}+1\right)$.

Finally, Eq. (B7) can be written:

$\theta_{C W}=\frac{2}{3} X \theta_{C u}+\frac{1}{6} X x_{3} x_{4} B$

with the following superexchange and double exchange contributions to $\theta_{C W}$, respectively: 


$$
\begin{aligned}
& \theta_{s e}=\frac{2}{3} X \theta_{C u}, \\
& \theta_{d e}=\frac{1}{6} X x_{3} x_{4} B .
\end{aligned}
$$

Eq. (B11) allows to estimate the mixed valence $\left(\mathrm{Cr}^{3+}, \mathrm{Cr}^{4+}\right)$ bandwidth, $W_{\mathrm{d}}$, of the $3 d t_{2 g}$ band from the relation [4]:

$W_{\mathrm{d}}=2 \mathrm{~B}$.

Using the Holland and Brown equations [40] we can calculate the superexchange integrals $J_{\mathrm{aa}}$ and $J_{\mathrm{ab}}$ for the first two coordination spheres, taking into account the estimated values of $\theta_{\text {se }}$ from Eq. (B12) and the experimental values of the Curie temperature $T_{\mathrm{C}}$.

$\theta_{\mathrm{se}}=5\left(3 \mathrm{~J}_{\mathrm{aa}}+18 \mathrm{~J}_{\mathrm{ab}}\right)$,

$T_{\mathrm{C}}=5\left(\mathrm{~J}_{\mathrm{aa}}-2 \mathrm{~J}_{\mathrm{ab}}\right)$.

The double exchange $b_{\mathrm{aa}}$ and $b_{\mathrm{ab}}$ integrals ${ }^{4}$ can be calculated from the equation:

$B=6 b_{\mathrm{aa}}+36 \mathrm{~b}_{\mathrm{ab}}$,

where $b_{\text {aa }}$ stands for the contribution from the 6 nearest neighbours and $b_{\mathrm{ab}}$ stands for the contributions from the 36 second nearest neighbours and from the Lotgering [5] considerations:

$b_{a a}=5 b_{\mathrm{ab}}$

The calculated magnetic parameters: $\theta_{\mathrm{se}}, \theta_{\mathrm{de}}, B, W_{\mathrm{d}}, J_{\mathrm{aa}}, J_{\mathrm{ab}}, J_{\mathrm{ab}}, b_{\mathrm{aa}}, b_{\mathrm{ab}}, J_{e f f}^{a a}, J_{e f f}^{a b}$ are collected in Table 3.

\section{References}

[1] J. Kanamori, J. Phys. Chem. Solids 10 (1959) p.87.

[2] P.K. Baltzer, P.J. Wojtowicz, M. Robbins and E. Lopatin, Phys. Rev. 151 
(1966) p.367.

[3] P.K. Baltzer, H.W. Lehmann and M. Robbins, Phys. Rev. Lett. 15 (1965) p.493.

[4] J. Krok, J. Spałek, S. Juszczyk and J. Warczewski, Phys. Rev. B 28 (1983) p.6499.

[5] F.K. Lotgering, in Proceedings of the International Conference on Magnetism, Nottingham, 1964 (Institute of Physics, London, 1965), p.533.

[6] F.K. Lotgering and R.P. Van Stapele, Solid State Commun. 5 (1967) p.143.

[7] C.Th. Hollander, G. Sawatzky and C. Haas, Solid State Commun. 15 (1974) p.747.

[8] O. Yamashita, Y. Yamaguchi, I. Nakatani, H. Watanabe and K. Masumoto, J. Phys. Soc. Japan 46 (1979) p.1145.

[9] M. Robbins, A. Menth, M.A. Miksovsky and R.C. Sherwood, J. Phys. Chem. Solids 31 (1970) p.423.

[10] T. Groń, A. Krajewski, J. Kusz, E. Malicka, I. Okońska-Kozłowska and A. Waśkowska, Phys. Rev. B 71 (2005) p.035208.

[11] T. Groń, A. Krajewski, H. Duda and P. Urbanowicz, Physica B 373 (2006) p.245.

[12] T. Groń, S. Mazur, H. Duda, J. Krok-Kowalski and E. Malicka, Physica B 391 (2007) p.371.

[13] T. Groń, S. Mazur, H. Duda, J. Krok-Kowalski and E. Maciążek, J. Alloys Compd. 467 (2009) p.112.

[14] S. Mazur, T. Groń and I. Jendrzejewska, J. Alloys Compd. 480 (2009) p.19. 
[15] N. Bloembergen and S. Wang, Phys. Rev. 93 (1954) p.72.

[16] I. Okońska-Kozłowska and J. Kopyczok, Acta. Cryst. C49 (1993) p.1448.

[17] CrysAlis CCD and CrysAlis RED, Oxford Diffraction Ltd., (2004) Wrocław, Poland.

[18] G.M. Sheldrick, "SHELXL-99, Program for the Refinement of Crystal Structures”, University of Göttingen, 1997.

[19] C. A. Bates, A. Gavaix, P. Steggles, A. Vasson and A. M. Vasson, J. Phys. C: Solid State Phys. 9 (1976) p.2413.

[20] A. Bencini and D. Gatteshi, Electron Paramagnetic Resonance of Exchange Coupled Systems, Springer-Verlag, Berlin Heidelberg, 1990.

[21] A. Abragam and B. Bleaney, Electron Paramagnetic Resonance of Transition Ions, Clarendon Press, Oxford, 1970.

[22] S.E. Barnes, Adv. Phys. 30 (1981) p.801.

[23] D.L. Huber and M.S. Seehra, J. Phys. Chem. Solids 36 (1975) p.723.

[24] M.S. Seehra, M.M. Ibrahim, V.S. Babu and G. Srinivasan, J. Phys.: Condens. Matter 8 (1996) p.11283.

[25] S.M. Bhagat and P. Lubitz, Phys. Rev. B 10 (1974) p.179.

[26] T. Kasuya, Progr. Theor. Phys. 22 (1959) p.227.

[27] H.J. Trodahl, Phys. Rev. B 51 (1995) p.6175.

[28] C. Kittel, Introduction to solid state physics, John Wiley \& Sons Inc., New York, 1966.

[29] J. Callaway, Quantum theory of the solid state, Academic Press, New York and London, 1974. 
[30] E. McCann and V.I. Fal'ko, J. Mag. Mag. Mater. 268 (2004) p.123.

[31] M. Matoba, S. Anzai and A. Fujimori, J. Phys. Soc. Jpn. 63 (1994) p.1429.

[32] M. Matoba, S. Anzai and A. Fujimori, J. Phys. Soc. Jpn. 60 (1991) p.4230.

[33] F.J. Blatt, P.A. Schroeder, C. Foiles and D. Greig, Thermoelectric power of metals, Plenum, New York, 1976.

[34] N.F. Mott, Conduction in non-crystalline materials, Oxford Univ. Press, New York, 1987.

[35] P.G. de Gennes, Phys. Rev. 118 (1960) p.141.

[36] P. Debye, Ann. Physik 39 (1912) p.789.

[37] Yu. Tretyakov, I.V. Gordeev and Ya.A. Kesler, J. Solid State Chem. 20 (1977) p.345.

[38] M. Cieplak, Phys. Rev. B 18 (1978) p.3470.

[39] J. Krok-Kowalski and J. Warczewski, J. Mag. Mag. Mater. 83 (1990) p.485.

[40] W.E. Holland and H.A. Brown, Phys. Status Solidi A 10 (1972) p.249. 
Table 1. Crystal data, experimental details and structure refinement results for the $\mathrm{CuCr}_{1.6} \mathrm{~V}_{0.4} \mathrm{Se}_{4}$ single crystal.

\section{(I) Crystal data and experimental details}

Structural formula

Space group

Lattice parameter $a(\mathrm{pm})$

Z

Calculated density $d\left(\mathrm{Mg} / \mathrm{m}^{3}\right)$

Crystal size $(\mathrm{mm} \times \mathrm{mm} \times \mathrm{mm})$

Maximum $2 \theta(\mathrm{deg})$

Index ranges: $\mathrm{h}$

$\mathrm{k}$

1

Reflections collected

Independent reflections

Data/parameters

Extinction correction

Final $R$ indices $[\mathrm{I}>2 \sigma(\mathrm{I})]: \mathrm{R}_{1} ; w \mathrm{R}_{2}$

Goodness of fit on $F^{2}$

Minimum and max $\Delta \rho\left(\mathrm{e}^{-3}\right)$
$(\mathrm{Cu})\left[\mathrm{Cr}_{1.6} \mathrm{~V}_{0.4}\right] \mathrm{Se}_{4}$

$F d \overline{3} m(\# 227)$

$1034.99(12)$

8

5.818

$0.13 \times 0.13 \times 0.14$

90.52

$-13,20$

$-20,16$

$-20,13$

5589

262

190/10

$0.0029(2)$

$2.79 ; 6.55$

1.009

$-2.57 ; 2.86$

(II) Fractional atomic coordinates and isotropic displacement parameters $\left(10^{3} \times \AA^{2}\right)$

$\begin{array}{lllllll}\text { Atom } & \text { Site } & \mathrm{x} & \mathrm{y} & \mathrm{z} & \mathrm{U}_{\text {iso }} & \text { Occupancy } \\ \mathrm{Cu} & 8 a & 0.125 & 0.125 & 0.125 & 9.2(1) & 1.0 \\ \mathrm{Cr} & 16 d & 0.5 & 0.5 & 0.5 & 10.4(2) & 0.8 \\ \mathrm{~V} & 16 d & 0.5 & 0.5 & 0.5 & 10.4(2) & 0.2 \\ \mathrm{Se} & 32 e & 0.25691(3) & 0.25691(3) & 0.25691(3) & 13.99(2) & 1.0\end{array}$


(V) Selected interatomic distances $(\AA)$ and bond angles (deg)
$\mathrm{Cu}-\mathrm{Se}(\mathrm{pm})$
236.47(5)
$\mathrm{Cr} / \mathrm{V}-\mathrm{Cu}(\mathrm{pm})$
429.08(5)
$\mathrm{Cr}-\mathrm{Se}(\mathrm{pm})$
251.80(3)
$\mathrm{Cr}-\mathrm{Cr}(\mathrm{pm})$
$365.92(4)$
Se - Se shortest $(\mathrm{pm})$
$345.70(8)$
$\mathrm{Se}-\mathrm{Se}(\mathrm{pm})$
$366.20(4)$
$\mathrm{Se}-\mathrm{Cu}-\mathrm{Se}(\mathrm{deg})$
109.47(0)
$\mathrm{Se}-\mathrm{Cr} / \mathrm{V}-\mathrm{Se}(\mathrm{deg})$
93.30(1)
$\mathrm{Se}-\mathrm{Cr}-\mathrm{Se}^{i}(\mathrm{deg})$
86.70(1)

Note: The superscript $i$ denotes symmetry operator generating equivalent atom at $\mathrm{x}+1 / 4$, $\mathrm{y}+1 / 4,1-\mathrm{z}$ 
Table 2. The fitting parameters: $D, E, F, G, H, I$ and $K$ in Eq. (4) of the thermoelectric power analysis of the $\mathrm{CuCr}_{1.6} \mathrm{~V}_{0.4} \mathrm{Se}_{4}$ single crystal.

\begin{tabular}{lllllllll}
\hline $\mathrm{D}$ & $\mathrm{E}$ & $\mathrm{F}$ & $\mathrm{G}$ & $\mathrm{H}$ & $\mathrm{I}$ & $\mathrm{K}$ & $\mathrm{R}$ & $\theta_{\mathrm{D}}$ \\
$\left(\mu \mathrm{V} / \mathrm{K}^{2}\right)$ & $\left(\mu \mathrm{V} / \mathrm{K}^{4}\right)$ & $(\mu \mathrm{V} / \mathrm{K})$ & $\left(10^{-3}\right)$ & $\left(\mu \mathrm{V} / \mathrm{K}^{1.5}\right)$ & $(\mu \mathrm{V} / \mathrm{K})$ & $\left(10^{-3}\right)$ & $\%$ & $(\mathrm{~K})$ \\
\hline 0.0227 & $1.3 \cdot 10^{-15}$ & 3.473 & 0.0054 & 0.0276 & 2.169 & 0.0071 & 99.3 & 279.7
\end{tabular}

$R$ is the agreement index. The Debye temperature $\theta_{D}$ has been calculated from Eq. (A1).

Table 3. The calculated magnetic parameters: $X, \theta_{\mathrm{se}}, \theta_{\mathrm{de}}, B, W_{\mathrm{d}}, J_{\mathrm{aa}}, J_{\mathrm{ab}}, b_{\mathrm{aa}}, b_{\mathrm{ab}}$, $J_{e f f}^{a a}$ and $J_{e f f}^{a b}$ of the $\mathrm{CuCr}_{2-2 \mathrm{x}} \mathrm{V}_{2 \mathrm{x}} \mathrm{Se}_{4}$ spinel system.

\begin{tabular}{|c|c|c|c|c|c|c|c|c|c|c|c|c|}
\hline $\begin{array}{l}\mathrm{x} \\
\left(\mathrm{V}^{3+}\right)\end{array}$ & $\begin{array}{l}\mathrm{x}_{3} \\
\left(\mathrm{Cr}^{3+}\right)\end{array}$ & $\begin{array}{l}\mathrm{x}_{4} \\
\left(\mathrm{Cr}^{4+}\right)\end{array}$ & $\mathrm{X}$ & $\begin{array}{l}\theta_{\mathrm{se}} \\
(\mathrm{K})\end{array}$ & $\begin{array}{l}\theta_{\mathrm{de}} \\
(\mathrm{K})\end{array}$ & $\begin{array}{l}\text { B } \\
(\mathrm{K})\end{array}$ & $\begin{array}{l}\mathrm{W}_{\mathrm{d}} \quad \mathrm{J}_{\mathrm{aa}} \\
(\mathrm{eV}) \quad(\mathrm{K})\end{array}$ & $\begin{array}{l}\mathrm{J}_{\mathrm{ab}} \\
(\mathrm{K})\end{array}$ & $\begin{array}{l}\mathrm{b}_{\mathrm{aa}} \\
(\mathrm{K})\end{array}$ & $\begin{array}{l}\mathrm{b}_{\mathrm{ab}} \\
(\mathrm{K})\end{array}$ & $\begin{array}{l}J_{e f f}^{a a} \\
(\mathrm{~K})\end{array}$ & $\begin{array}{l}J_{e f f}^{a b} \\
(\mathrm{~K})\end{array}$ \\
\hline 0 & 0.512 & 0.488 & 2.896 & 90 & 340 & 4391 & $0.76 \quad 63.9$ & -9.65 & 332.65 & 65.53 & 466.51 & -83.05 \\
\hline 0.2 & 0.41 & 0.39 & 2.718 & 163 & 44 & 607 & $0.11 \quad 31.7$ & -3.47 & 45.98 & 9.2 & 197.55 & -38.7 \\
\hline
\end{tabular}

The values for $x=0.0$ are taken from Ref. 13 for comparison. 
Figure captions:

Figure 1. Electrical resistivity $\rho$ and thermoelectric power $S$ vs. temperature $T$.

Figure 2. Magnetoresistance $\Delta \rho / \rho_{0}$ vs. magnetic induction B at $4.3,10,15,20$ and $40 \mathrm{~K}$.

Figure 3. Temperature dependences of mass susceptibility $\chi_{\sigma}$ and reciprocal mass susceptibility $\chi_{\sigma}^{-1}$ recorded at $H=900$ Oe. The Curie temperature $T_{\mathrm{C}}=$ 193.2 $\mathrm{K}$ is indicated by arrow.

Figure 4. Mass magnetisation $\sigma$ vs. magnetic field $H$ at $4.6 \mathrm{~K} . \sigma_{\mathrm{s}}=53.84$ Oe. $\mathrm{cm}^{3} / \mathrm{g}$ and $\mu_{\mathrm{s}}=4.66 \mu_{\mathrm{B}}$ are the saturation magnetisation and saturation magnetic moment, respectively. The saturation state is marked by dashed line. Inset: the onset of the $\sigma(\mathrm{H})$ dependence up to $1.0 \mathrm{kOe}$.

Figure 5. The temperature dependence of the ESR parameters: (a) the resonance linewidth $\triangle B$, (b) the resonance field $B_{\mathrm{r}}$ and (c) intensity of the spectrum DI. The Curie temperature $T_{\mathrm{C}}=193.8 \mathrm{~K}$ is indicated by arrow and separates FMR and EPR resonances. 
Figure 6. Model of paramagnetic relaxation processes of the $\mathrm{CuCr}_{1.6} \mathrm{~V}_{0.4} \mathrm{Se}_{4}$ spinel system.

Figure 7. The thermopower analysis of the $\mathrm{CuCr}_{1.6} \mathrm{~V}_{0.4} \mathrm{Se}_{4}$ spinel where $S_{\text {exp }}$ is the experimental curve, $S_{\text {theor }}$ is the theoretical curve and $S_{\text {diff, }} S_{p h}, S_{\text {imp }}$ and $S_{\text {mag }}$ are diffusion, phonon drag, impurity and magnon drag components of thermopower, respectively. 


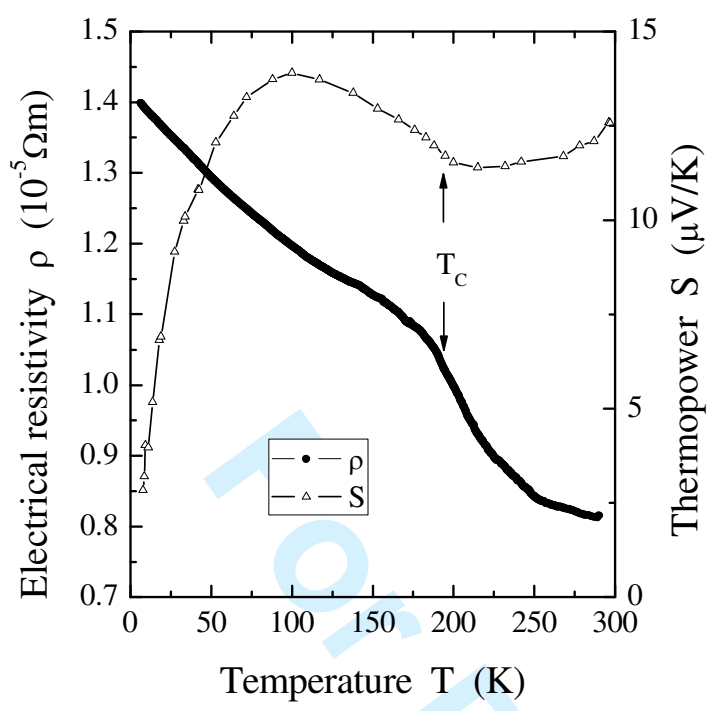

Fig. 1



Fig. 2 




Fig.3

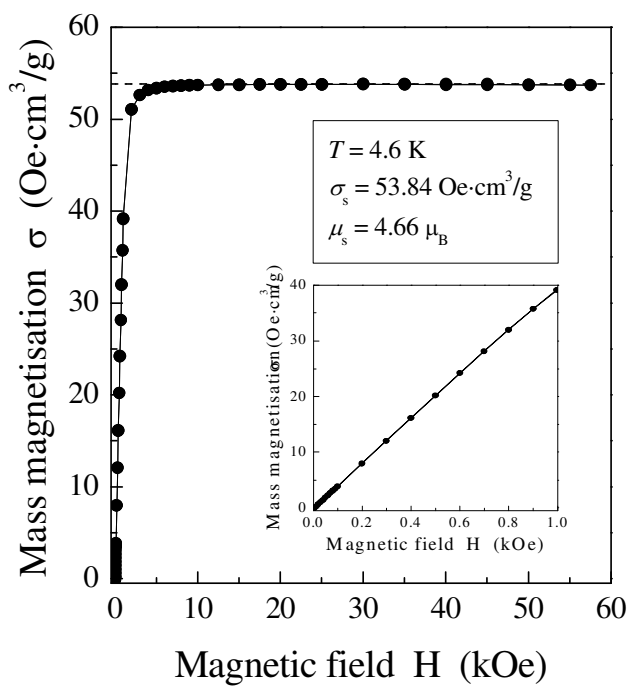

Fig.4 




Fig.5

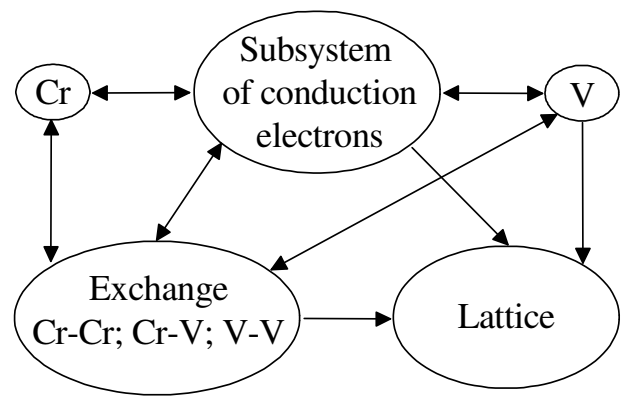

Fig.6 


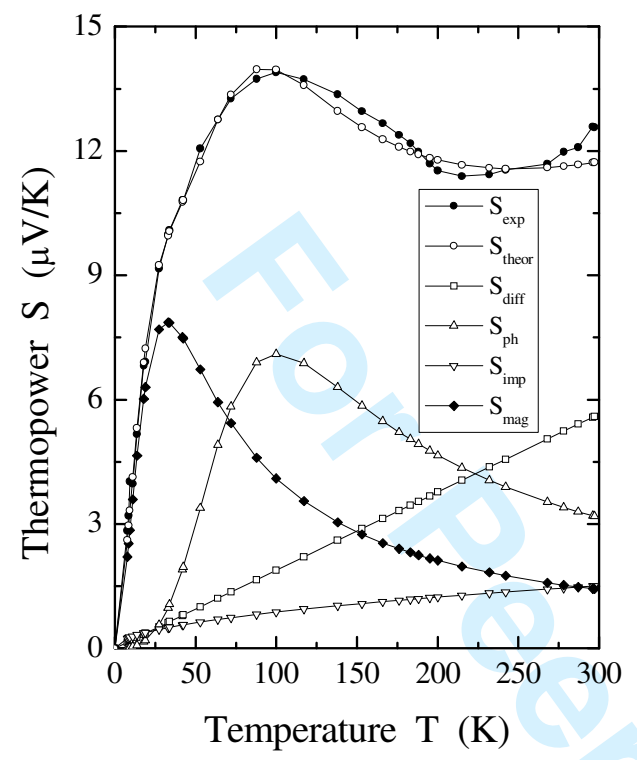

Fig.7 